\title{
Processes required for the assurance of quality results of a measurement standard
}

\section{Alexei PIANîH,}

National Institute for Metrology, Head of mass laboratory and masses derivates,

e-mail: mase@metrologie.md, tel.: (+373) 22903139

Summary: This work characterizes the logical processes required to be carried out with a measuring instrument to assure quality results. As an example, we shall analyze pressure measuring instruments. The aim is to confirm the reliability of the measurement capability, respectively, its relevance. We shall focus on the description of processes that should be maintained on the given equipment.

Key words: processes, standard, control chart, competence, calibration, maintenance, intermediate check, calibration interval.

\section{INTRODUCTION}

Pressure unit is a derivative measurement unit of the International System of Units [1]. Within the mass laboratory and masses derivates (laboratory), pressure is reproduced mostly using pressure balance, which represent pressure measurement instruments (MI), frequent used as standard due to their special performances. Because of the fact that pressure measurements imply a wide diversification (depending on the environment, measurement interval, functioning principle etc.), as working standards, may also be used calibrators, indicators, pressure micromanovacuummeters. So, the system of standards of the National Institute for Metrology (INM) confirms its utility. The main purpose is taking over the pressure measurement unit, its conservation and dissemination to the reference standards, and, thus, assuring uniformity of measurements performed on the territory of the Republic of Moldova, inclusive within the economic, technical and scientific relations with other countries.

Measurement capabilities of laboratory cover most metrological requirements of the measurement instruments used in the Republic of Moldova. Figure 1 presents the metrological traceability scheme of the pressure measurement unit.

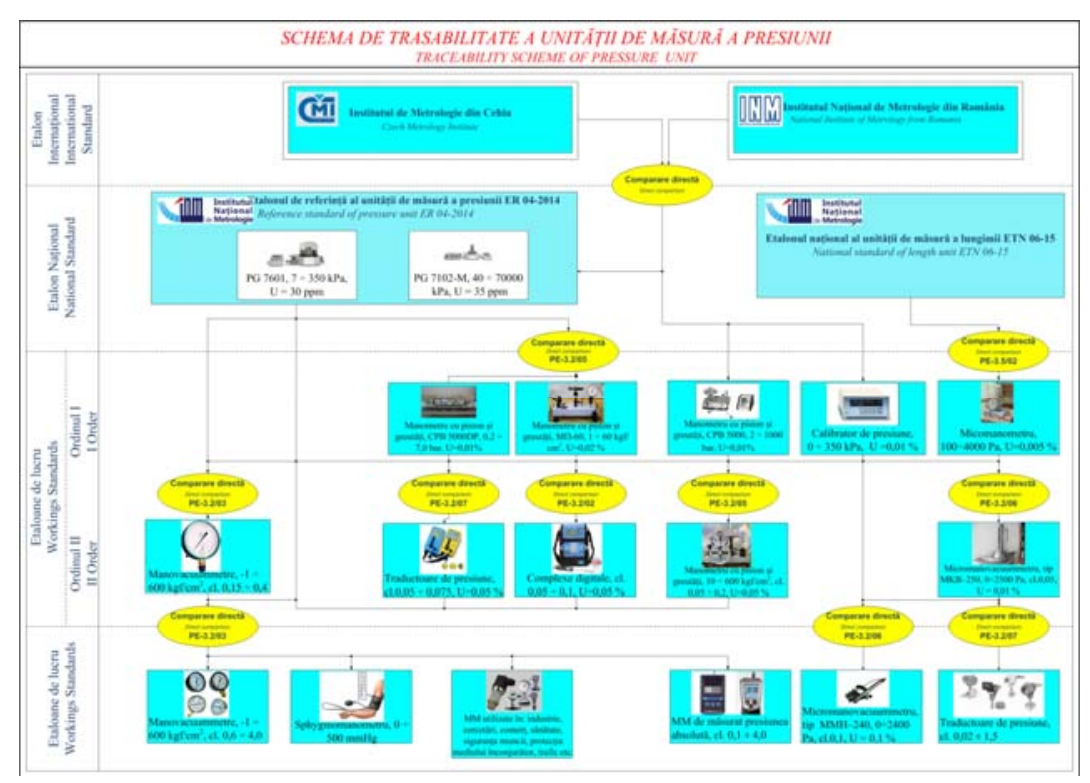

Figure 1. Metrological traceability scheme of the pressure measurement unit

\section{PROCESSES REQUIRED FOR THE ASSURANCE OF QUALITY RESULTS OF A MEASUREMENT STANDARD}

The Quality Management System (QMS) implies the analysis of all components that can influence the measurement process. [2]. The processes are monitored so that the result of the measurement process to be within the reasonable and satisfying expectance limits or, at least, clear for the beneficiary. Nevertheless, as seen in practice, it is difficult for the laboratory staff to structure the periodicity of the processes required to be performed with the 
standard, as well as the proofs relevant for an audit. Thus, this article shall present an example of observing the objective interferences for the assurance of quality results reproduced by a measurement standard.

We shall classify the processes that influence the result depending on their impact. So, there are two main categories, as seen in Figure 2:

Macro-processes - intercomparisons, calibrations, control charts, replicated calibrations. Macro-processes represent actions undertaken for observing the standard continuously, during a long period of time. These are mainly focused on the assurance of proficiency evidences and require the compliance with related standards/guides, as well as the cooperation with other institutions.

Micro-processes - intermediate check, maintenance, inspection before and after transportation. Micro-processes represent actions undertaken for observing the standard instantaneously and after every change in its status. These are mainly focused on the confirmation of the manufacturer's recommendations, QMS requirements and require a highly competent staff involved in the work.

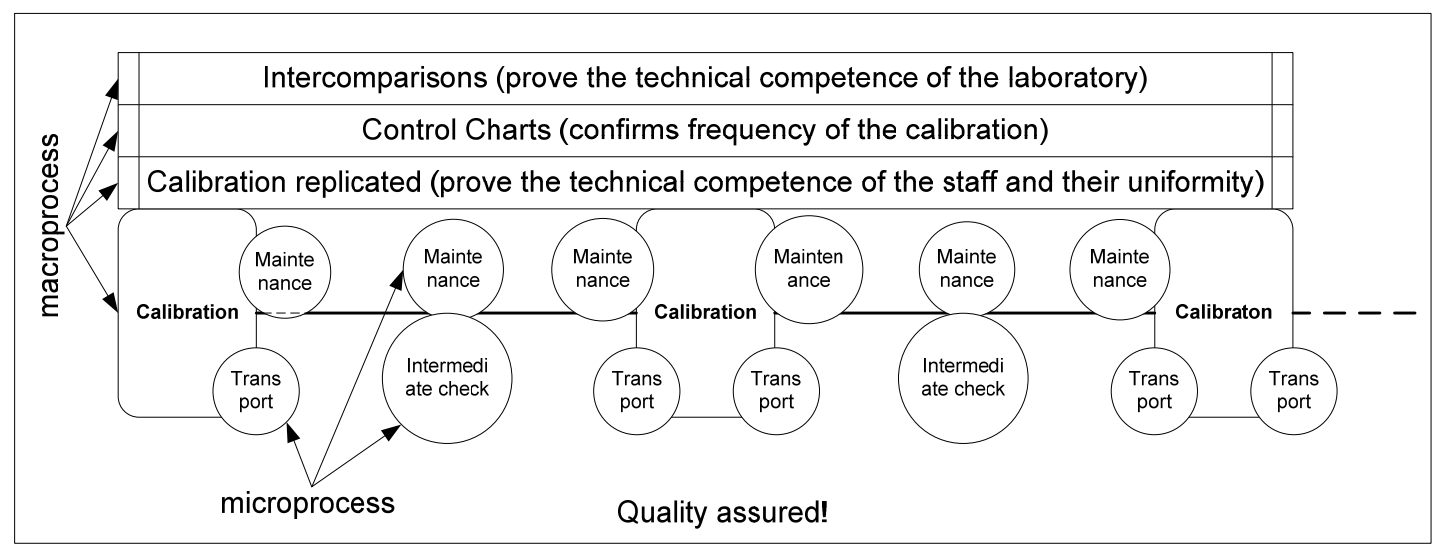

Figure 2. Optimal distribution of processes for quality assurance

\section{Intercomparison}

This process has the purpose of confirming the measurement capabilities of testing/calibration and metrological verification laboratories. Laboratories that prove their measurement capabilities - Figure 3 - have the assurance of the reliability of their measurements.

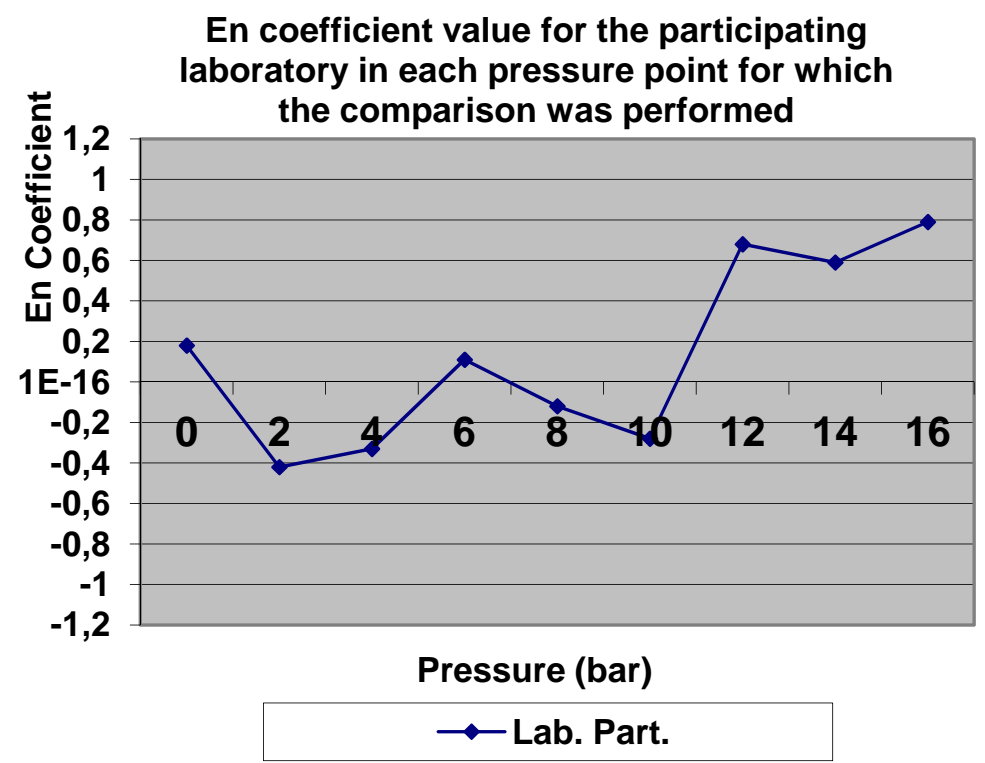

Figure 3 Real results obtained by laboratory of INM within the intercomparison Clab 11:2016 


\section{Calibration}

Activity that establishes, in certain conditions: - firstly, a relation between the values and related measurement uncertainties provided by standards and the readouts in accordance with the related measurement uncertainties; - secondly, this information is used to establish a relationship that would allow obtaining a measurement result starting from a readout [3].

The metrological traceability of a measurement result can be confirmed only through calibration.

\section{Control chart}

Control charts, like other results of researches on exploitation risks, recalibration of $\mathrm{MI}$, and improvements and/or tendencies observed in time, are introduced in the technical fiches of $\mathrm{MI}$ as research reports.

The performed analyses may include information regarding the calibration interval, periodicity of standard use, measurement unit drift, descriptions of observations and improvements. The results may serve as basis for reasoning the modification of the maintenance and calibration program of the National Measurement Standards Database.

Figure 4 presents an example of a control chart of a pressure balance calibrated already for 3 times. It can be observed that the main metrological characteristic - effective area of the piston - has a linear stability in time and is within the acceptable error limits.

The 5 years frequency of calibration and stability of ER 04:2014 is confirmed [4], [5].
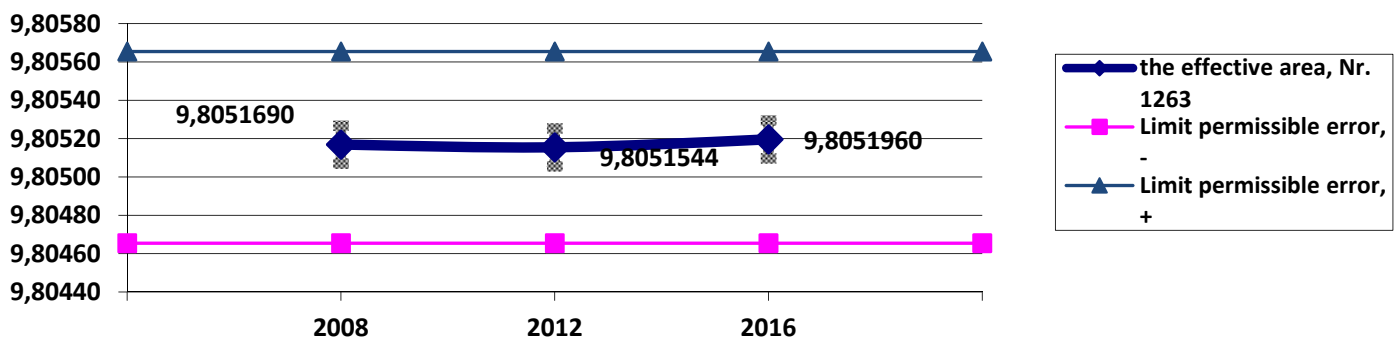

Figure 4. Control chart presenting the stability of the effective area of the piston no. 1263

$\left(x-\right.$ years, $\left.y-\mathrm{cm}^{2}\right)$, part of the type PG 7601 no. 663 weight piston manometer

\section{Calibration replicated}

Calibration replicated has the purpose of validating a calibration method used by a laboratory or the proficiency of staff to use a calibration procedure, for the uniformity of the result. For a clear presentation, Figure 5 shows the types of replicated calibrations. When calibrating a MI through two different methods, one being standardized, the validation of the method described in the calibration procedure is aimed. When calibrating a $\mathrm{MI}$ through one single method, but by two engineers or using two different standards, the confirmation of the staff proficiency is aimed.

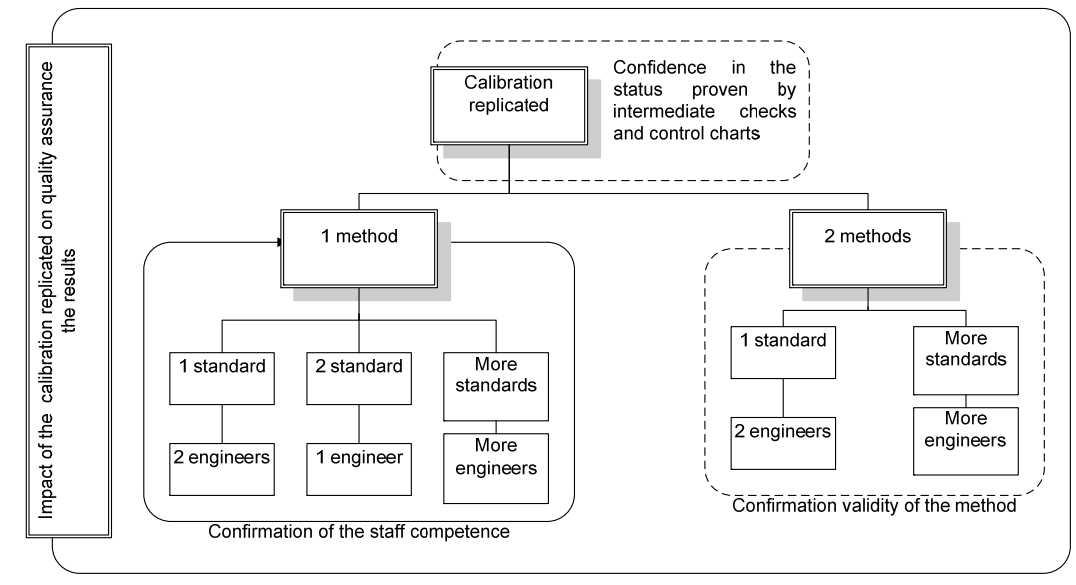

Figure 5. Calibration replicated performance flow chart 
For example, in the case of the pressure indicator RPM4 A350Ks BA100Ks from laboratory, calibrated at Metas, Switzerland in 2016 using a similar PG 7601 standard, owned by INM, Republic of Moldova, there were repeated the measurements at the same points, through the same method. Table 1 presents the measurements' results.

Table 1. Values obtained from the calibration certificate no. $133-07555 / 2016$, issued by METAS, Switzerland on 15.06.2016 and the replicated calibration no. 024-3.2/2016, issued by laboratory, INM on 07.09.2016

\begin{tabular}{|c|c|c|c|c|c|}
\hline \multirow{2}{*}{$\begin{array}{c}\text { Conventional } \\
\text { value, kPa }\end{array}$} & \multicolumn{2}{|c|}{ Readout, kPa } & \multicolumn{2}{c|}{$\begin{array}{r}\text { Expanded uncertainty, } \\
\mathrm{kPa}\end{array}$} & \multirow{2}{*}{ En } \\
\cline { 2 - 5 } & METAS & INM & METAS & INM & \\
\hline 100 & $-0,002$ & $-0,006$ & 0,0050 & 0,06 & 0,08 \\
\hline 125 & $-0,001$ & $-0,014$ & 0,0075 & 0,06 & 0,21 \\
\hline 150 & 0,000 & $-0,010$ & 0,0075 & 0,06 & 0,16 \\
\hline 200 & 0,000 & $-0,013$ & 0,0075 & 0,06 & 0,20 \\
\hline 250 & 0,000 & $-0,013$ & 0,0075 & 0,06 & 0,21 \\
\hline 300 & 0,001 & $-0,007$ & 0,0075 & 0,06 & 0,13 \\
\hline 350 & 0,005 & $-0,008$ & 0,0075 & 0,06 & 0,21 \\
\hline
\end{tabular}

Due to the fact that the normalized errors are lower than 1,0 it was confirmed that the quality of measurements obtained using the pressure indicator RPM4 A350Ks BA100Ks, no. 920 and the weight piston manometer PG 7601, no. 1263 are compatible with those reproduced by our colleagues from Switzerland, as well as the staff proficiency.

\section{Transportation}

This process has the purpose of immediate inspection of the $\mathrm{Ml}$ before and after transportation, when the standard's location is changed. This activity assures the lack of deviations from the normal status. The conditions that have to be fulfilled when moving a MI are, normally, described in instructions or manuals of the equipment, received from the manufacturer. In case these instructions are absent, the general safety rules, described in the MI technical manual, apply.

The general actions to be fulfilled when transporting mechanical and electronic pressure measurement devices are:

- The correctness of MI functioning when connecting and disconnecting it from the power source;

- The control of the presence of all parts of the equipment and their careful use (with special gloves, if necessary).

This micro-process is usually unpleasant for the engineers, because it implies a QMS monitoring also. But the inspection process of the Ml status is very important, as it allows a quick reaction to the change of the standard's normal status, and it can help the laboratory save on rejection costs.

\section{Intermediate check}

Intermediate check has the purpose of maintaining confidence in standards or in the equipment's calibration condition. The confirmation or invalidation of the validity of the most recent calibration certificate of the standard is aimed.

This monitoring implies the inspection of the physical condition of the standard, equivalent to the control of the exterior aspect (lack of deviations from the previous condition). This control assures the confidence in the main technical/metrological characteristics of the standard. 


\section{Maintenance}

Maintenance has the purpose of maintaining the functionality of the $\mathrm{MI}$, by observing its behavior in real time of the standard and assuring its stability, through the performance of certain activities described by the manufacturer.

The real processes that represent the maintenance are:

- cleaning and/or washing surfaces of dust, oil, dirt (cleaning the oil is done with ethylic alcohol, on all parts of the equipment);

- monitoring the presence of lubricants, the satisfying level of liquids, gases or reference materials;

- changing the work environment;

- adjusting the zero reference, the verticality or other technical conditions;

- polishing metal surfaces;

- applying paint;

- eliminating/introducing a gas/liquid in the system;

- treating some surfaces with technical vaseline etc.

\section{CONCLUSIONS}

The processes described in this article represent an example of observing the reliability of a standard's results quality. Arranging the work processes for a standard is a difficult task, but very important. At first sight, they seem to be confusing, but after a deep and professional analysis, they can be arranged accordingly and logically. Once the processes are systematized, they stop being a discomfort for the engineer and are performed more efficient.

Based on the equipment from the laboratory, relevant examples were presented regarding the way processes, meant to assure measurement results quality, are realized and appreciated. But we have to take into account the fact that the given examples are not regulated by any standard, and represent the result of INM research. They can be used as starting point or best practice for the quality infrastructure laboratories that want to implement an efficient QMS.

\section{BIBLIOGRAPHY}

[1] http://www.bipm.org/en/measurement-units/base-units.html

[2] SM SR EN ISO/CEI 17025:2006 "General requirements for the competence of testing and calibration laboratories"

[3] pct. 2.39 SM SR Ghid ISO/CEI 99:2012 "Vocabulary of Metrology. Basic and General Concepts and Associated Terms (VIM)"

[4] Guideline DKD-R 6-1 "Calibration of Pressure Gauges"

[5] OIML D 10 2007/ ILAC-G24 2007 "Guidelines for the determination of calibration intervals of measuring instruments" http://www.iec-ilac-iaf.org/doc/1007a.pdf 